\title{
Oral Manifestation of Paraneoplastic Pemphigus
}

\author{
Seurin Kim, In Hee Park, YounJung Park, Jeong-Seung Kwon, Jong-hoon Choi, Hyung-Joon Ahn \\ Department of Orofacial Pain and Oral Medicine, Dental Hospital of Yonsei University College of Dentistry, Seoul, Korea
}

Received June 14, 2019

Revised July 9, 2019

Accepted July 9, 2019

Correspondence to:

Hyung-Joon Ahn

Department of Orofacial Pain and Oral

Medicine, Dental Hospital of Yonsei

University College of Dentistry, 50-1 Yonsei-

ro, Seodaemun-gu, Seoul 03722, Korea

Tel: +82-2-2228-3113

Fax: +82-2-393-5673

E-mail: HJAHN@yuhs.ac

https://orcid.org/0000-0001-9669-9781
Paraneoplastic pemphigus (PNP) is a rare and often fatal autoimmune blistering disease accompanied by both benign and malignant neoplasms. Usually, oral, skin, and mucosal lesions are the earliest manifestations shown by PNP patients. Oral ulcers are initial lesions in various autoimmune diseases like pemphigus, bullous pemphigoid, erythema multiforme, graft-versus-host, lichen planus, it does not improved despite of high-dose steroid therapy. We report a-35-year-old female who presented oral ulceration, lip crust and skin lesions. By doing several examinations, such as enzyme-linked immunosorbent assay, incisional biopsy with indirect immunofluorescence, she was diagnosed PNP with non-Hodgkin's lymphoma on pancreas.

Key Words: Enzyme-linked immunosorbent assay; Fluorescent antibody technique, indirect; Oral ulcer; Pemphigus

\section{INTRODUCTION}

Paraneoplastic pemphigus (PNP) is a rare, autoimmune mucocutaneous blistering disease, frequently associated with lymphoproliferative disorders [1]. These disorders are typically chronic lymphocytic leukemia, lymphoma, Castleman's disease, and thymoma. PNP was first reported by Anhalt et al. [2] in 1990. PNP accounts for 3\% to 5\% of all pemphigus cases. Without any significant difference between male and female, it arises usually in patients aged between 45 and 70 years [3]. PNP can affect also children and adolescents. In this group of patients, PNP is more frequently associated with Castleman's disease and hematologic malignant disorders [4].

The usual initial manifestation is painful progressive stomatitis. PNP lesions exists not only the oral mucosa, but also esophagus, stomach, duodenum, and colon [5]. It is characterized by painful mucosal erosions and polymorphic cutaneous lesions [6]. Erythema multiforme (EM)-like or lichenoid eruptions, rather than blister-like lesions, are more commonly observed [7].
PNP has been considered as more resistant to medical therapies in comparison to other forms of pemphigus [8]. Corticosteroids are considered as first selected medication. However, these only improve the skin lesion, while mucosal lesion is not affected by steroid [9]. We document a case of PNP in a-35-year-old female who suffers from non-Hodgkin's lymphoma.

\section{CASE REPORT}

A-35-year-old female presented to the Department of Orofacial Pain and Oral Medicine, Dental Hospital of Yonsei University (Seoul, Korea) complaining of painful oral ulceration happened 4 months ago (Fig. 1). The patient also had skin lesions on hands, feet, arms (Fig. 2), and genital lesion. Cutaneous eruptions occurred following mucosal lesions. The patient had extensive and painful oral ulceration with crust and spots of bleeding on lip, and multiple skin lesions with no pain and itching. However, the severity of skin lesions was much less than the oral ulcers.

Although she had already taken nonsteroidal anti-inflam- 


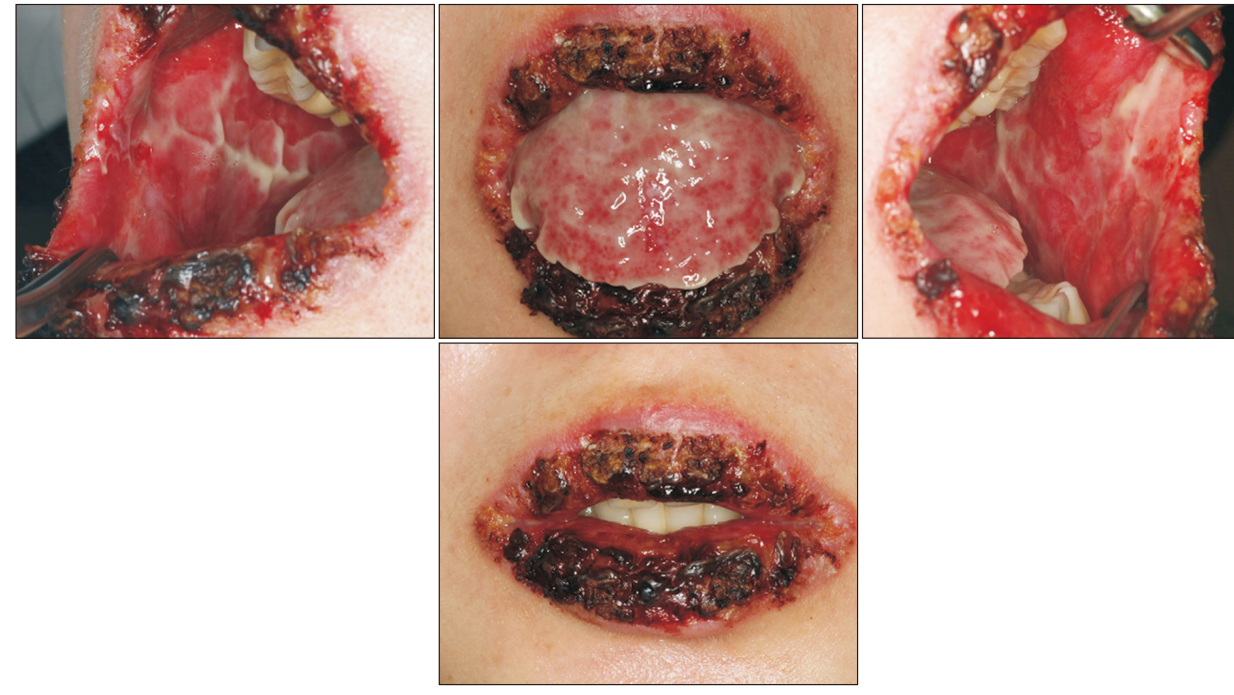

Fig. 1. Oral and lip lesions at the first visit.

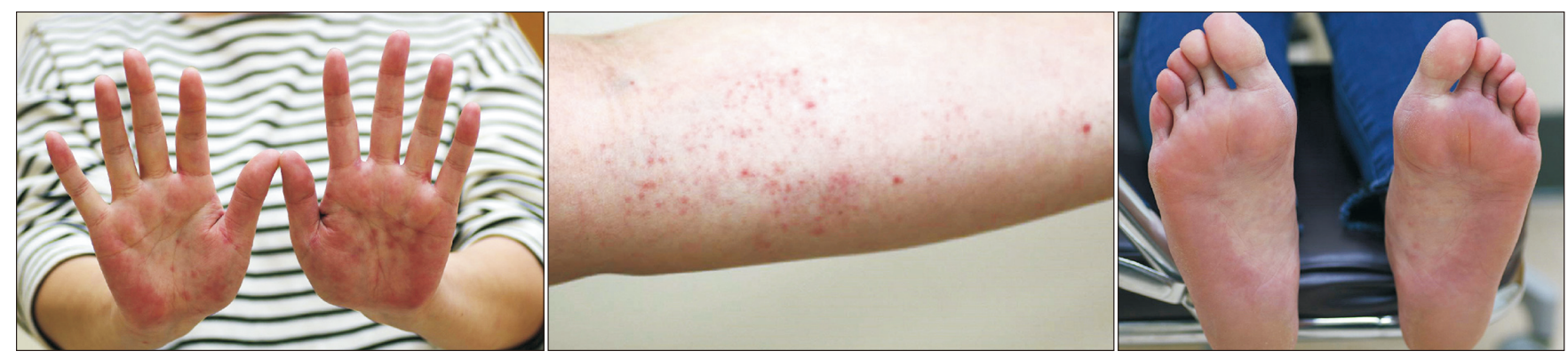

Fig. 2. Skin lesions at the first visit.

matory drugs and steroid prescribed from other hospitals, her condition was remained. In previous serological test by other hospitals, antinuclear antibody and human leukocyte antigen B51 were positive, these mean the possibility that she has autoimmune diseases or Behcet's disease.

In our department, Human immunodeficiency virus and Hepatitis C virus test (OraQuick; OraSure Technologies, Inc., Bethlehem, PA, USA) was done and the result was negative. Additional serological test was done, including erythrocyte sedimentation rate, C-reactive protein, Vitamin B12, folate, Zinc, anti-desmoglein 1 Enzyme-linked immunosorbent assay (ELISA), anti-desmoglein 3 ELISA, Herpes simplex virus antibody (IgG, IgM). In addition, she was prescribed topical and systemic prednisolone, and consulted to dermatology for skin lesion evaluation

In department of the dermatology, an incisional biopsy was performed for histology. The histopathology revealed lichen planus (LP), showing interface dermatitis with basal vacuolization. At that time, the result of serum anti-desmoglein 3 ELISA which was done by our department at first visit is positive, that means the possibility of pemphigus. She visited our department, again. Although she took medicine properly and applied ointment, there is no improvement (Fig. 3).

These various clinical, serological, and histological results stand for the possibility of PNP. Also, Indirect immunofluorescence (IIF) of the patient's serum on rat bladder substrate showed markedly positive staining, a finding strongly associated with PNP with a reported specificity of 83\% to $100 \%$. Thus, an underlying malignancy was sought, despite there being no symptoms or features on physical examination to suggest such a diagnosis

For the evaluation, chest, abdomen, pelvis computerized tomography (CT) were taken. CT shows splenomegaly with enhanced masses, possibly lymphoproliferative disease, such as lymphoma or Castleman's disease (Fig. 4).

For further evaluation, she was consulted to the department of hemato-oncology. Bone marrow biopsy did not 

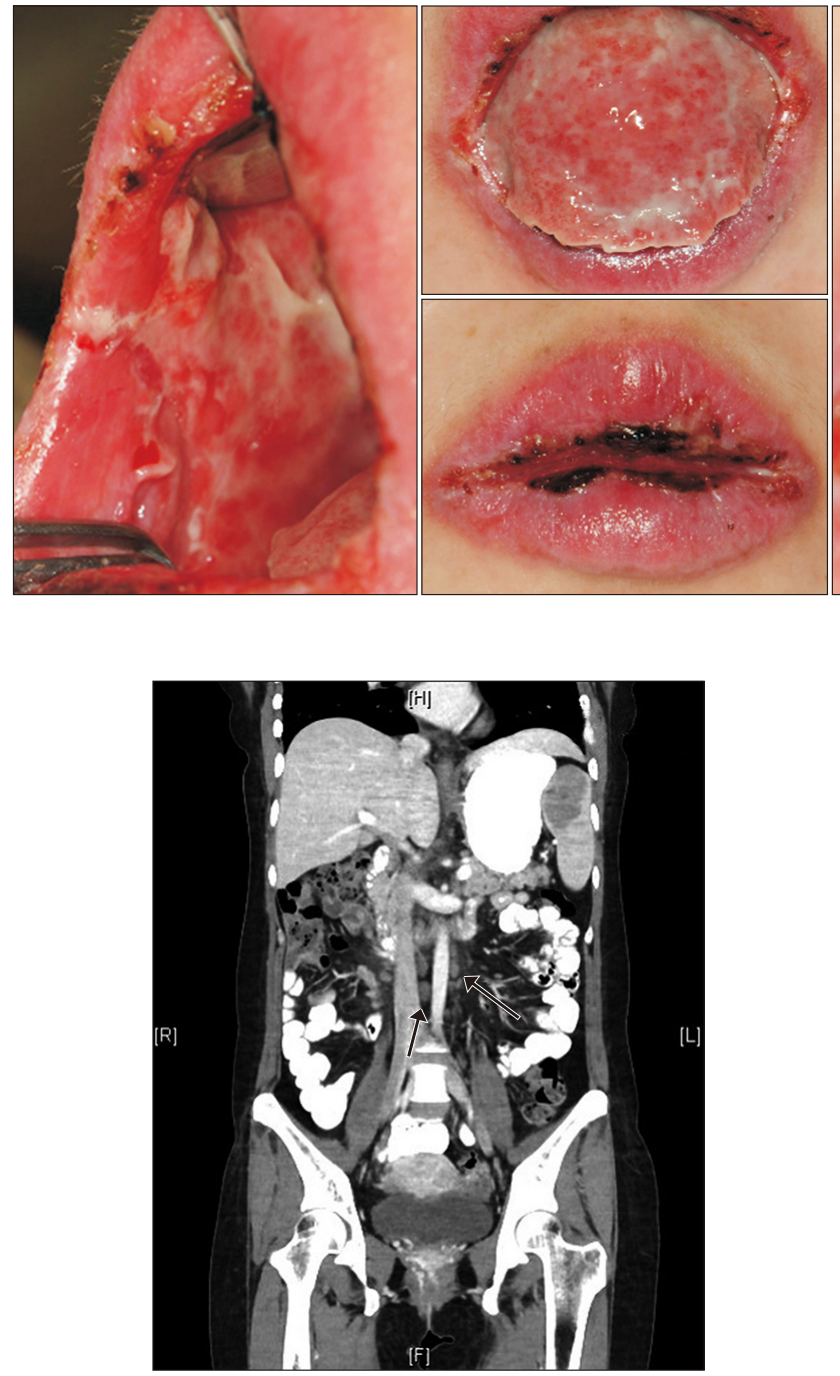

Fig. 4. The chest, abdomen, pelvis computerized tomography image, showing spenomegaly with multiple homogeneously enhanced masses (arrows).

reveal any abnormalities. Splenectomy was performed followed by excision for histology as the cytology was suggestive of a malignancy CD20+ indolent B cell non-Hodgkin lymphoma. The histopathology and immunohistochemistry confirmed that as a non-Hodgkin's lymphoma. She has no family history. No metastases were identified.

\section{DISCUSSION}

PNP is associated with benign and malignant neoplasm, such as non-hodgkin's lymphoma, chronic lymphocytic leukemia, and Castleman's disease. A 3\% to 5\% of all pemphigus cases are diagnosed with PNP. The age range of

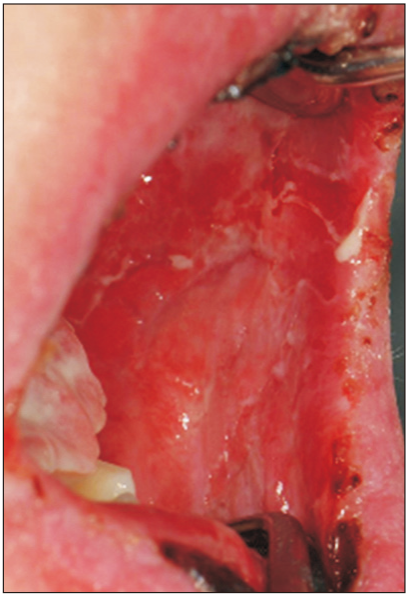

Fig. 3. Intraoral photo at the second visit.

affected individuals is usually from 45 to 70 years without gender difference. In about 30\% of all PNP, the first clinical manifestation leads to the detection of an occult tumor [3].

Clinical features of PNP are extremely polymorphous. PNP lesions can be detected not only on skin, but also in different mucosal area [2]. Especially, oral mucosa is almost always involved and usually the vermillion border of the lips is included, which resembles the oral lesion of EM [10]. Ulceration may involve the entire oral mucosal surface, such as painful stomatitis caused by massive erosion in the oropharynx [11]. Usually, skin lesions appear after the onset of mucosal lesions [12]. A single patient may present different types of lesions, each of which is able to evolve from one type to another [3,12]. Usually, oral and cutaneous lesions of PNP resemble those seen in pemphigus vulgaris, bullous pemphigoid, EM, graft versus host disease (GVHD) or LP $[8,13]$.

The pathogenesis of PNP is not yet completely understood. One of the proposed pathogenesis is that lymphoid neoplasm causes immune dysregulation, leading to autoantibody production against self-antigens [14]. A subsequent theory focuses on the initial role of desmoglein 1 and desmoglein 3, which are autoantigens in clinical variants of pemphigus. In addition, PNP produces autoantibodies against proteins of the plakin and cadherin families, which are involved in cell structure maintenance and tissue cohesion. As structural components of desmosome and hemidesmosome, their autoantibody reaction in keratin cytoskeleton of keratinocytes can result in the suprabasal clefting in PNP [15]. 
The diagnostic criteria for PNP was first proposed by Anhalt et al. [2] with the introduction of the disease. It includes mucocutaneous blistering and ulceration as well as histopathological features, such as acantholytic changes of the epithelium and epidermis with interface dermatitis, deposition of IgG and C3 in intercellular areas and/or along the basement membrane, presence of various desmoplakins and desmogleins in the serum which can be identified by ELISA. An important histological finding to diagnosis PNP is dyskeratosis with suprabasal acantholysis [3]. However, sometimes the acantholysis is difficult to find, even though it is important diagnostic pitfalls with other diseases as EM, Stevens Johnson Syndrom, GVHD, and drug reactions [16]. Serological testing for PNP is based primarily on the detection of anti-plakin autoantibodies including desmoplakin, periplakin, and envoplakin. ELISA is a useful tool to detect anti-desmoglein 3 and anti-desmoglein 1 autoantibodies in PNP, although PNP patients usually show only anti-desmoglein 3 antibody [17]. Direct immunofluorescence (DIF) result usually shows weak or moderate positivity in the intercellular area for IgG and C3 [2]. IIF identifies autoantibodies directed against plakins, among which autoantibodies to envoplakin and periplakin are the most specific. IIF on rat bladder is now thought as a useful screening test for PNP due to the high specificity (83\%) [18].

High-dose corticosteroids are still considered as a first line therapy of PNP [9]. PNP therapy remains challenging because of the rarity of the disease. Although several medical therapies have been suggested in the literature, PNP has been considered to be more resistant to medical therapies in comparison to other forms of pemphigus [19].

Early diagnosis of PNP is critical. When patient clinically presents severe and refractory oral mucosal ulceration or heterogeneous oral lesion including pemphigus, pemphigoid, LP, and EM, we should consider the possibility of PNP. Further evaluation should include incisional biopsy with DIF and anti-desmoglein ELISA. When the patient shows certain features include intercellular substance and basement membrane staining on DIF, and suprabasal acantholysis, keratinocyte necrosis and lichenoid infiltration on histopathology [1,2], clinicians should consider PNP, even if the patient is otherwise systemically sound.

In addition, we should carefully examine the patient who is diagnosed with pemphigus with heterogeneous clinical and/or histologic findings. Furthermore, diagnosis and management of PNP requires close collaboration between physicians, including dermatologist, oncologist, and dentist. Since the oral lesion of the PNP occurs in its initial stage, the role of dental clinicians is significant in diagnosis of PNP.

\section{CONFLICT OF INTEREST}

No potential conflict of interest relevant to this article was reported

\section{ACKNOWLEDGEMENTS}

This work was supported by the National Research Foundation of Korea (NRF) grant funded by the Korea government (MIST) (no. 2016R1A5A2008630).

\section{ORCID}

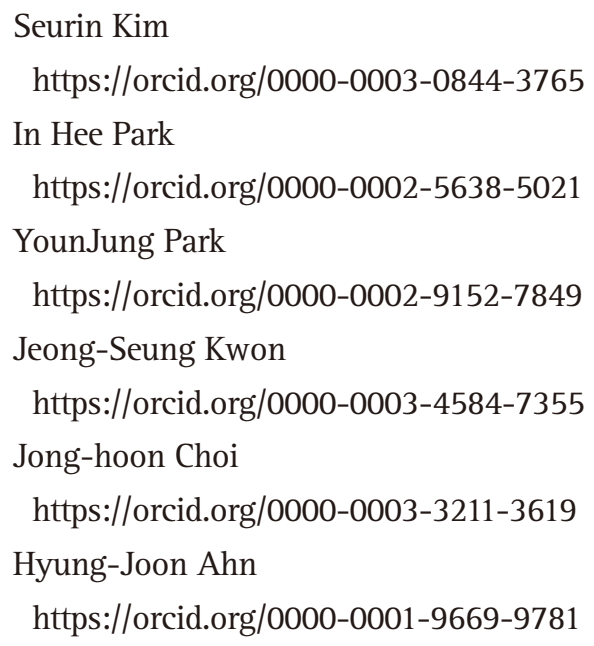

\section{REFERENCES}

1. Zimmermann J, Bahmer F, Rose C, Zillikens D, Schmidt E. Clinical and immunopathological spectrum of paraneoplastic pemphigus. J Dtsch Dermatol Ges 2010;8:598-606.

2. Anhalt GJ, Kim SC, Stanley JR, et al. Paraneoplastic pemphigus. An autoimmune mucocutaneous disease associated with neoplasia. N Engl J Med 1990;323:1729-1735.

3. Vassileva S, Drenovska K, Manuelyan K. Autoimmune blistering dermatoses as systemic diseases. Clin Dermatol 2014;32:364-375.

4. Mimouni D, Anhalt GJ, Lazarova Z, et al. Paraneoplastic pem- 
phigus in children and adolescents. Br J Dermatol 2002;147:725732.

5. Porro AM, Caetano Lde V, Maehara Lde S, Enokihara MM. Nonclassical forms of pemphigus: pemphigus herpetiformis, IgA pemphigus, paraneoplastic pemphigus and IgG/IgA pemphigus. An Bras Dermatol 2014;89:96-106.

6. Helm TN, Camisa C, Valenzuela R, Allen CM. Paraneoplastic pemphigus. A distinct autoimmune vesiculobullous disorder associated with neoplasia. Oral Surg Oral Med Oral Pathol 1993;75:209213.

7. Choi Y, Nam KH, Lee JB, et al. Retrospective analysis of 12 Korean patients with paraneoplastic pemphigus. J Dermatol 2012;39: 973-981.

8. Yong AA, Tey HL. Paraneoplastic pemphigus. Australas J Dermatol 2013;54:241-250.

9. Gergely L, Váróczy L, Vadász G, Remenyik E, Illés A. Successful treatment of B cell chronic lymphocytic leukemia-associated severe paraneoplastic pemphigus with cyclosporin A. Acta Haematol 2003;109:202-205.

10. Paolino G, Didona D, Magliulo G, et al. Paraneoplastic pemphigus: insight into the autoimmune pathogenesis, clinical features and therapy. Int J Mol Sci 2017;18:E2532.

11. Kelly S, Schifter M, Fulcher DA, Lin MW. Paraneoplastic pemphigus: two cases of intra-abdominal malignancy presenting solely as treatment refractory oral ulceration. J Dermatol 2015;42:300-
304.

12. Zhu X, Zhang B. Paraneoplastic pemphigus. J Dermatol 2007;34: 503-511.

13. Frew JW, Murrell DF. Paraneoplastic pemphigus (paraneoplastic autoimmune multiorgan syndrome): clinical presentations and pathogenesis. Dermatol Clin 2011;29:419-425, viii.

14. Kartan S, Shi VY, Clark AK, Chan LS. Paraneoplastic pemphigus and autoimmune blistering diseases associated with neoplasm: characteristics, diagnosis, associated neoplasms, proposed pathogenesis, treatment. Am J Clin Dermatol 2017;18:105-126.

15. Hertl M, Eming R, Veldman C. T cell control in autoimmune bullous skin disorders. J Clin Invest 2006;116:1159-1166.

16. Bialy-Golan A, Brenner S, Anhalt GJ. Paraneoplastic pemphigus: oral involvement as the sole manifestation. Acta Derm Venereol 1996;76:253-254.

17. Ishii N, Maeyama $\mathrm{Y}$, Karashima T, et al. Immunoserological analyses of 55 patients with pemphigus at the Dermatological Department of Kurume University Hospital: an 11-year retrospective study (1996-2006). Int J Dermatol 2008;47:1321-1322.

18. Helou J, Allbritton J, Anhalt GJ. Accuracy of indirect immunofluorescence testing in the diagnosis of paraneoplastic pemphigus. $\mathrm{J}$ Am Acad Dermatol 1995;32:441-447.

19. Lee SE, Hashimoto T, Kim SC. No mucosal involvement in a patient with paraneoplastic pemphigus associated with thymoma and myasthenia gravis. Br J Dermatol 2008;159:986-988. 